\title{
Association of Acarbose with Decreased Muscle Mass and Function in Patients with Type 2 Diabetes: A Retrospective, Cross-Sectional Study
}

Lan-lan Jiang · Xiao-hua Xu • Meng-hui Luo · Hui-ying Wang •

Bo Ding · Reng-na Yan · Yun $\mathrm{Hu} \cdot$ Jian-hua Ma (D)

Received: July 12, 2021 / Accepted: September 2, 2021 / Published online: September 20, 2021

(C) The Author(s) 2021

\section{ABSTRACT}

Introduction: Prevalence of sarcopenia has increased in patients with type 2 diabetes. The influence of glucose-lowering drugs on muscles in these patients remains unclear. We aimed to investigate the association between muscle mass/function and glucose-lowering drugs.

Methods: Data of 1042 hospitalized patients with type 2 diabetes were included in this retrospective, cross-sectional study. All the patients had stable hypoglycemic therapy in the last 3 months, and performed bioelectrical impedance analysis, grip strength, and gait speed tests on admission.

Results: Skeletal muscle index [6.81 (95\% CI $6.67,6.94)$ vs. $\left.7.17(7.09,7.24) \mathrm{kg} / \mathrm{m}^{2}\right]$,

Lan-lan Jiang and Xiao-hua Xu contributed equally to this article.

Supplementary Information The online version contains supplementary material available at https:// doi.org/10.1007/s13300-021-01151-6.

L. Jiang $\cdot$ X. Xu $\cdot$ M. Luo $\cdot$ H. Wang $\cdot$ B. Ding

R. Yan $\cdot$ Y. Hu $\cdot$ J. Ma $(\bowtie)$

Department of Endocrinology, Nanjing First

Hospital, Nanjing Medical University, No. 32

Gongqingtuan Road, Nanjing 210012, Jiangsu,

China

e-mail: majianhua196503@126.com

Y. $\mathrm{Hu}$

e-mail: huyunwuxi@163.com handgrip strength $[23.41(22.24,24.58)$ vs. $26.93(26.33,27.54) \mathrm{kg}]$, and gait speed [1.19 $(1.15,1.22)$ vs. $1.27(1.25,1.28) \mathrm{m} / \mathrm{s}]$ decreased in patients using acarbose compared with the others (all $p<0.001$ ). Gait speed and skeletal muscle index remained lower in patients using acarbose compared to their matched patients in propensity score matching $(p=0.036$ and 0.010 , respectively). Among drug-naïve patients and patients using insulin, metformin, sulfonylureas, or acarbose monotherapy, the acarbose group had lowest skeletal muscle index and handgrip strength $\left[6.81(6.52,7.11) \mathrm{kg} / \mathrm{m}^{2}\right.$ and $22.54(19.28,25.79) \mathrm{kg}, p=0.028$ and 0.001 , respectively].

Conclusion: Acarbose treatment was associated with decreased muscle mass and strength. Assessment and exercise of muscles in patients with long-term acarbose treatment should be considered.

Keywords: Muscles; Acarbose; Diabetes mellitus; Sarcopenia 


\section{Key Summary Points}

Why carry out this study?

The deterioration of skeletal muscle mass and function impairs physical function and increases health burden and costs.

Prevalence of sarcopenia increases in patients with type 2 diabetes, but the influence of glucose-lowering drugs on muscles remains unclear.

What was learned from the study?

Acarbose therapy, rather than insulin, metformin, sulfonylureas, and dipeptidyl peptidase- 4 inhibitors, was associated with both decreased muscle mass and function.

Assessment and exercise of muscles in patients with long-term acarbose treatment should be considered, and the mechanism and effects of acarbose on muscles need further study.

\section{INTRODUCTION}

Muscle mass and strength are critical determinants a person's quality of life. The deterioration of skeletal muscle mass and function caused by aging (sarcopenia) has grave physiological and clinical consequences, such as physical disability, bone fracture, depression, hospitalization, and even mortality [1-4]. Therefore, sarcopenia increases health burden and costs [5].

Patients with type 2 diabetes (T2DM) are at a higher risk of developing sarcopenia [6]. According to the Asian Working Group for Sarcopenia (AWGS) definition, the prevalence of sarcopenia is about $13.3-27.4 \%$ in older patients with T2DM in Asia, and is significantly higher than that in subjects without diabetes [7-11]. There is a bidirectional relationship between T2DM and sarcopenia [6]: since skeletal muscle consumes the most glucose in vivo, sarcopenia may contribute to the development and progression of T2DM; on the other hand, insulin resistance, inflammation, accumulation of advanced glycation end-products (AGEs), and increased oxidative stress in T2DM can negatively affect muscle mass and function.

Possible mechanisms of the high prevalence of sarcopenia in patients with T2DM include insulin resistance, inflammation and oxidative stress caused by hyperglycemia, microvascular and macrovascular complications $[6,12]$. Sustained hyperglycemia and oxidative stress in T2DM can lead to the accumulation of AGEs in muscles, which contributes to poor muscle health [13]. Recently, studies have begun to focus on the effects of glucose-lowering drugs on sarcopenia. In theory, glucose-lowering drugs can reduce blood glucose and thus prevent oxidative stress [14] and accumulation of AGEs [15] in patients with T2DM. The use of glucose-lowering drugs can also reduce the incidence of chronic microvascular and macrovascular complications [16]. Moreover, some glucose-lowering drugs, such as metformin and thiazolidinediones (TZDs), have effects on improvement of insulin resistance [17-19]. These effects may protect the patients with T2DM from sarcopenia. However, glucagon-like peptide 1 receptor agonists (GLP-1RAs), metformin, and sodium-glucose co-transporter 2 inhibitors (SGLT2i) decrease body weight by reducing energy intake or increasing glucose output [16]. This might accelerate agerelated loss of muscle [20]. Other drugs, such as insulin and sulfonylureas, may increase body weight and insulin resistance [16]. Therefore, the effects of most glucose-lowering drugs on muscle mass and strength remain unclear or controversial [6, 21-23], and there is no approved pharmacological treatment for sarcopenia that we aware of.

Although sarcopenia is age-related and usually assessed in old patients (age $\geq 60$ or 65 years), previous studies showed that the lean body mass as a percentage of total body weight begins to reduce from the third decade, and consequently, sarcopenia may develop in the young and middle-aged population [24, 25], 
and the difference of muscle mass among obesity, prediabetes, or diabetes already exists in young and middle-aged patients [26]. Therefore, without age limit, we analyzed the muscle mass, handgrip strength, and gait speed in patients with T2DM using different glucose-lowering drugs retrospectively in the present study. Our primary objective was to investigate the muscle mass and function in patients using different glucose-lowering drugs; and the secondary objectives include comparison of the muscle mass and function among drug-naïve patients and patients using insulin, metformin, sulfonylureas, or acarbose monotherapy, and the rate and characteristics of sarcopenia in patients with T2DM.

\section{METHODS}

\section{Study Design}

This was a retrospective, cross-sectional study, and was approved by the Institutional Ethical Committee of Nanjing First Hospital, Nanjing Medical University (20160201-01). The methods were conducted in accordance with the Declaration of Helsinki guidelines, including any relevant details. The need for informed consent was waived for the present study as the research involves no more than minimal risk to subjects, and the waiver did not adversely affect the rights and welfare of the subjects. All data was retrospectively collected from the medical review database without involving any identifiable private information under the consent of the corresponding department. Two researchers extracted data from consecutive medical records of patients admitted to the endocrinology department of Nanjing First Hospital. Data analysis covered the period from May 2019 to April 2020. The inclusion criteria were as follows: (1) patients who were diagnosed with T2DM; (2) patients who have not been treated with any hypoglycemic drugs (drug naïve) or there are no changes in glucose-lowering drugs in the last 3 months before the end of index date; (3) patients who finished the bioelectrical impedance analysis (BIA), grip strength, and step speed tests on admission. Patients were excluded if they (1) had acute infection; (2) had acute complications of diabetes on admission, such as ketoacidosis and lactic acidosis; (3) had severe systemic diseases or any other conditions that may influence muscle mass and function, such as disuse muscle atrophy, malignant tumor, severe cognitive disorder, and autoimmune disease.

Our sample size calculation was based on our previous cross-sectional study [27]. Acarbose was used by 32 of 248 patients. We also tested SMI in these patients, and the SMI level in patients using acarbose was $6.8 \mathrm{~kg} / \mathrm{m}^{2}$, whereas it was $7.2 \mathrm{~kg} / \mathrm{m}^{2}$ in the others. The standard deviation of SMI was $1.1 \mathrm{~kg} / \mathrm{m}^{2}$ in these patients. We needed at least 780 patients with $80 \%$ power and an $\alpha$ of 0.05 (assuming $10 \%$ data missing). The calculation was performed at http://www.biomath.info/power/index.html.

\section{Clinical and Laboratory Assessments}

Patient data regarding height, weight, age, duration of diabetes, hypoglycemic treatment, and other medication use were collected at the time of hospital admission. Concomitant diseases and diabetic complications, such as hypertension, fatty liver, atherosclerosis, diabetic kidney disease, neuropathy, retinopathy [28], osteoporosis, and low bone mass [29], were diagnosed by clinicians according to the Chinese guidelines for related diseases, and were recorded. Body mass index (BMI) was calculated as weight divided by the square of height $(\mathrm{kg} /$ $\mathrm{m}^{2}$ ). Blood samples of all patients were collected after overnight fasting (for more than $10 \mathrm{~h}$ ). Glycosylated hemoglobin (HbA1c) was measured using high-performance liquid chromatography assay (Bio-Rad Laboratories, Inc. CA, USA). C-peptide was measured using chemiluminescent immunometric assay which employs the Modular Analytics E170 (Roche ${ }^{\circledR}$ Diagnostics GmbH, Mannheim, Germany). Insulin resistance was evaluated using Homeostasis Model Assessment 2-Insulin Resistance (HOMA2-IR), which was calculated using fasting C-peptide and blood glucose by HOMA2 Calculator [30]. 
Table 1 Clinical characteristics

\begin{tabular}{|c|c|c|c|c|}
\hline & \multirow[t]{2}{*}{ Total population } & \multicolumn{2}{|c|}{ Elderly patients (age $\geq 60$ years) } & \multirow[t]{2}{*}{$p$ value $^{*}$} \\
\hline & & Sarcopenia & Non-sarcopenia & \\
\hline Number & 1042 & 141 & 350 & \\
\hline Age & $58.19(57.41,58.97)$ & $71.33(70.18,72.47)$ & $67.73(67.11,68.36)$ & $<0.001$ \\
\hline Gender (male \%) & $631(60.56)$ & $78(55.32)$ & $193(55.14)$ & 1.000 \\
\hline Drinking (\%) & $107(10.27)$ & $8(5.67)$ & $30(8.57)$ & 0.352 \\
\hline Current smoking (\%) & $203(19.48)$ & $12(8.51)$ & $57(16.29)$ & 0.031 \\
\hline Family history (\%) & $273(26.20)$ & $16(11.35)$ & $79(22.57)$ & 0.004 \\
\hline Diabetic duration (year) & $7.67(7.25,8.09)$ & $10.42(9.08,11.77)$ & $9.77(8.97,10.57)$ & 0.413 \\
\hline Height $(\mathrm{cm})$ & $165.39(164.88,165.89)$ & $160.74(159.51,161.97)$ & $164.31(163.45,165.17)$ & $<0.001$ \\
\hline Weight (kg) & $68.17(67.36,68.97)$ & $56.66(55.28,58.04)$ & $68.09(67.06,69.13)$ & $<0.001$ \\
\hline $\operatorname{BMI}\left(\mathrm{kg} / \mathrm{m}^{2}\right)$ & $24.8(24.58,25.03)$ & $21.9(21.45,22.36)$ & $25.21(24.88,25.54)$ & $<0.001$ \\
\hline Waist to hip ratio & $0.91(0.91,0.92)$ & $0.9(0.89,0.91)$ & $0.92(0.91,0.92)$ & 0.001 \\
\hline Handgrip strength $(\mathrm{kg})$ & $26.23(25.68,26.77)$ & $19.89(18.79,21)$ & $24.68(23.80,25.56)$ & $<0.001$ \\
\hline Gait speed $(\mathrm{m} / \mathrm{s})$ & $1.25(1.24,1.26)$ & $1.08(1.04,1.12)$ & $1.21(1.19,1.23)$ & $<0.001$ \\
\hline $\operatorname{SMI}\left(\mathrm{kg} / \mathrm{m}^{2}\right)$ & $7.09(7.03,7.16)$ & $6.01(5.89,6.13)$ & $7.11(7.02,7.20)$ & $<0.001$ \\
\hline Systolic pressure ( $\mathrm{mmHg}$ ) & $128.51(127.53,129.48)$ & $129.7(127.15,132.26)$ & $130.89(129.2,132.59)$ & 0.686 \\
\hline Heart rate $(/ \mathrm{min})$ & $77.11(76.63,77.59)$ & $77.26(75.31,79.21)$ & $76.20(75.39,77.00)$ & 0.248 \\
\hline Albumin $(\mathrm{g} / \mathrm{L})$ & $39.98(39.76,40.21)$ & $38.79(38.33,39.25)$ & $39.57(39.17,39.98)$ & 0.011 \\
\hline $\operatorname{ALT}(\mathrm{U} / \mathrm{L})$ & $28.54(27.2,29.88)$ & $24.45(21.45,27.45)$ & $26.15(24.17,28.13)$ & 0.066 \\
\hline $\operatorname{AST}(\mathrm{U} / \mathrm{L})$ & $20.04(19.12,20.96)$ & $18.25(16.57,19.93)$ & $19.71(18.21,21.21)$ & 0.403 \\
\hline Total cholesterol $(\mathrm{mmol} / \mathrm{L})$ & $4.49(4.42,4.56)$ & $4.07(3.89,4.26)$ & $4.37(4.24,4.49)$ & 0.011 \\
\hline Triglyceride (mmol/L) & $2.60(1.90,3.3)$ & $1.48(1.27,1.68)$ & $2.18(1.50,2.87)$ & 0.010 \\
\hline LDL-c (mmol/L) & $1.93(1.89,1.97)$ & $1.67(1.56,1.78)$ & $1.87(1.80,1.94)$ & 0.002 \\
\hline $\mathrm{HDL}-\mathrm{c}(\mathrm{mmol} / \mathrm{L})$ & $1.21(1.19,1.23)$ & $1.27(1.22,1.33)$ & $1.26(1.21,1.30)$ & 0.244 \\
\hline Calcium $(\mathrm{mmol} / \mathrm{L})$ & $2.27(2.26,2.28)$ & $2.25(2.23,2.27)$ & $2.27(2.26,2.28)$ & 0.075 \\
\hline Phosphate (mmol/L) & $1.19(1.18,1.20)$ & $1.15(1.12,1.19)$ & $1.16(1.14,1.18)$ & 0.559 \\
\hline Creatinine $(\mu \mathrm{mol} / \mathrm{L})$ & $67.70(66.26,69.14)$ & $66.29(61.95,70.63)$ & $71.25(68.75,73.74)$ & 0.004 \\
\hline Uric acid $(\mu \mathrm{mol} / \mathrm{L})$ & $309.44(301.44,317.45)$ & $320.05(278.21,361.89)$ & $314.61(303.22,325.99)$ & 0.169 \\
\hline White blood cell $\left(\times 10^{9}\right)$ & $6.00(5.89,6.11)$ & $5.59(5.21,5.97)$ & $6.03(5.82,6.23)$ & 0.062 \\
\hline Red blood cell $\left(\times 10^{12}\right)$ & $4.42(4.38,4.45)$ & $4.18(4.10,4.26)$ & $4.34(4.29,4.40)$ & 0.007 \\
\hline Hemoglobin $(\mathrm{g} / \mathrm{L})$ & $137.02(134.95,139.09)$ & $129.21(126.83,131.59)$ & $133.91(132.11,135.71)$ & 0.003 \\
\hline $\mathrm{FBG}(\mathrm{mmol} / \mathrm{L})$ & $8.67(8.41,8.93)$ & $8.14(7.61,8.66)$ & $8.49(8.15,8.84)$ & 0.257 \\
\hline PBG (mmol/L) & $15.89(15.36,16.42)$ & $15.17(13.8,16.55)$ & $16.28(15.39,17.17)$ & 0.195 \\
\hline
\end{tabular}


Table 1 continued

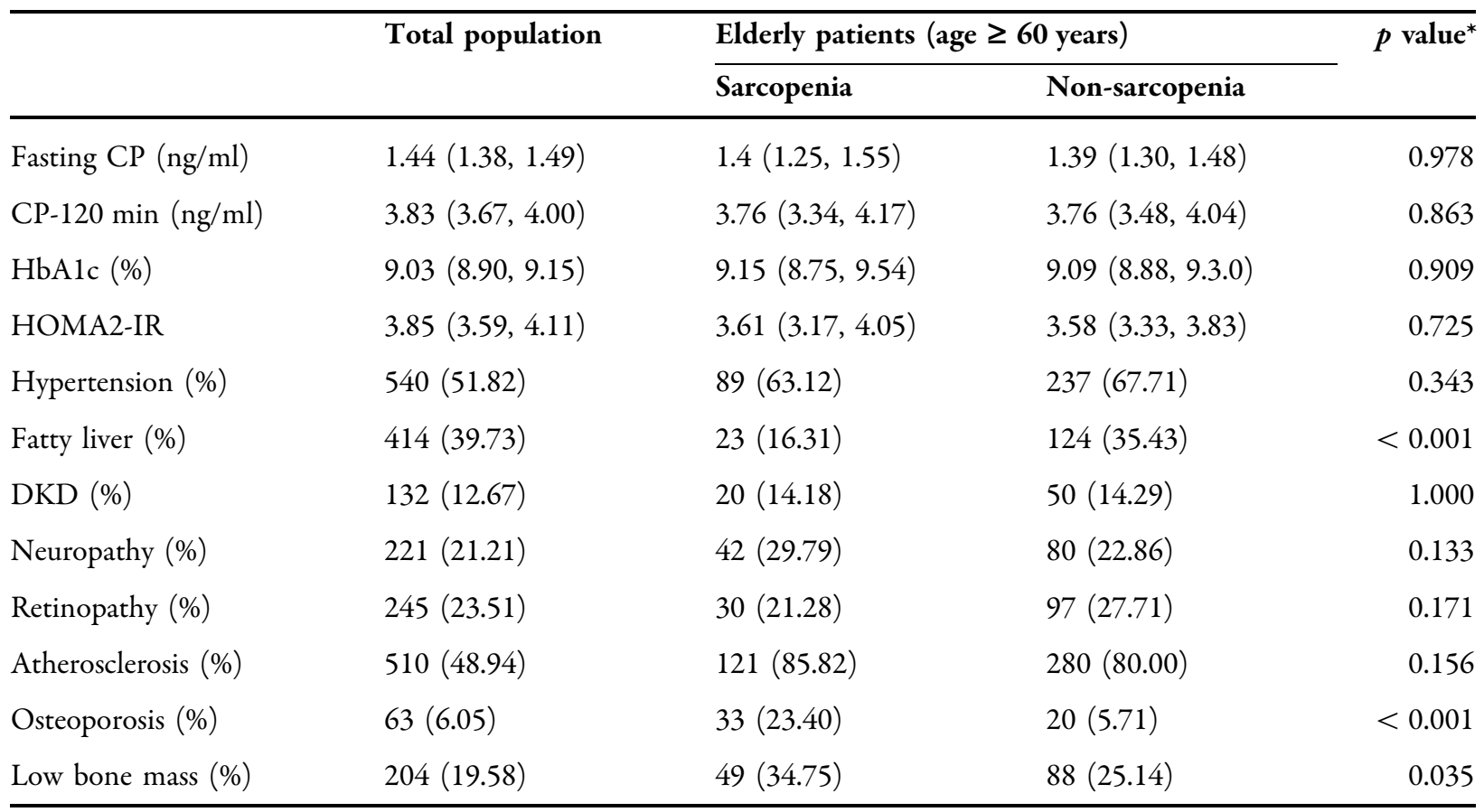

$B M I$ body mass index, $S M I$ skeletal muscle index, $A L T$ alanine transferase, $A S T$ aspartate transferase, $L D L-c$ low density lipoprotein cholesterol, $H D L-c$ high density lipoprotein cholesterol, $F B G$ fasting blood glucose, $P B G$ postprandial blood glucose, CP C-peptide, HbA1c hemoglobin, HOMA2-IR Homeostasis Model Assessment 2-Insulin Resistance, DKD diabetic kidney disease

${ }^{*}$ Sarcopenia vs. non-sarcopenia

\section{Muscle Mass and Function Assessment}

Skeletal muscle mass (SMM) was tested by multifrequency BIA (InBody 770 body composition analyzer, Korea). Skeletal muscle index $(\mathrm{SMI})=\mathrm{SMM} /$ height $^{2} \times 100 \%$. Muscle strength was tested using the handgrip strength measurement, and taking the maximum reading of at least two trials using both hands in a maximum-effort isometric contraction. Physical performance was assessed using six-meter gait speed. Sarcopenia was diagnosed according to AWGS (2019) when there was low muscle mass (defined as skeletal muscle index $<7.0 \mathrm{~kg} / \mathrm{m}^{2}$ in men and $<5.7 \mathrm{~kg} / \mathrm{m}^{2}$ in women), together with either low muscle strength (defined as handgrip strength $<28 \mathrm{~kg}$ in men and $<18 \mathrm{~kg}$ in women) or low physical performance (defined as six-meter gait speed $<1.0 \mathrm{~m} / \mathrm{s}$ ) or both [31].

\section{Statistical Analysis}

Statistical analyses were performed using the SPSS software (SPSS, Science, Chicago, USA). All variables were tested for normal distribution. Data are presented as means (95\% CI). Propensity score matching was used to adjust confounders of effects of glucose-lowering drugs on muscles. Differences between the different two groups were examined using Student s unpaired $t$ test for parametric data or the Mann-Whitney $U$ test for non-parametric data, respectively. The differences among different glucose-lowering drugs were analyzed using Kruskal-Wallis test. 
The categorical data were examined with chisquare test. All comparisons were two-sided at a $5 \%$ significance level. A $p$ value less than 0.05 was considered to be statistically significant.

\section{RESULTS}

\section{Study Populations and Clinical Characteristics}

A total of 1077 patients with T2DM completed the tests for sarcopenia. Among these patients, 35 patients were excluded because of severe hepatic impairment or kidney disease, hyperthyroidism, infection, or heart failure, and 1042 patients were finally enrolled for analysis. Fiftyfive patients lacking data for HbA1c were still included for analysis. There were 491 patients with age $\geq 60$ years. Among these old patients, 141 (28.7\%) had sarcopenia, 154 (31.4\%) patients had muscle mass reduction (SMI $<7.0 \mathrm{~kg} / \mathrm{m}^{2}$ in men and $<5.7 \mathrm{~kg} / \mathrm{m}^{2}$ in women), $257(52.3 \%)$ patients had low muscle strength, and 93 (18.9\%) had low physical performance. The clinical characteristics of the total population and elderly patients with or without sarcopenia are shown in Table 1 . The patients with sarcopenia had lower height, weight, BMI, waist to hip ratio, albumin, blood lipids, creatinine, red blood cell count, and hemoglobin levels than the other elderly patients (all $p<0.05$ ). Moreover, the sarcopenia patients were older, and had lower rates of current smoking, diabetes family history, fatty liver, and had higher rates of osteoporosis than the non-sarcopenia patients (all $p<0.05$ ). However, blood glucose and HOMA2-IR were similar between the two groups (both $p>0.05$ ).

\section{Muscle Mass, Strength, and Physical Performance in Patients Using Different Glucose-Lowering Drugs}

SMI, handgrip strength, and gait speed in patients using insulin $(n=287)$, metformin $(n=365)$, sulfonylureas $(n=259)$, acarbose $(n=215)$, dipeptidyl peptidase 4 (DPP4) inhibitors $(n=104)$, TZDs $(n=26)$, glinides $(n=40)$,
GLP-1RAs $(n=22)$, and SGLT2 inhibitors $(n=40)$ were respectively compared to those in the patients who were not using the drug (including the drug-naïve patients and the patients using other glucose-lowering drugs). SMI [6.81 $(95 \%$ CI $6.67,6.94)$ vs. $\left.7.17(7.09,7.24) \mathrm{kg} / \mathrm{m}^{2}\right]$, handgrip strength $[23.41(22.24,24.58)$ vs. $26.93(26.33,27.54) \mathrm{kg}]$, and gait speed [1.19 $(1.15,1.22)$ vs. $1.27(1.25,1.28) \mathrm{m} / \mathrm{s}]$ decreased in patients using acarbose compared with the others (all $p<0.001$ ). Acarbose was associated with lower SMI, handgrip strength, and gait speed in both men and women (all $p<0.05$, Fig. 1). Patients using DPP4 inhibitors had lower SMI than the others $(p<0.05$, Fig. 1A, B). Patients using insulin therapy had lower handgrip strength compared with the others $(p<0.05$, Fig. 1C, D), as well as the gait speed in men $(p<0.05$, Fig. 1E). Men using TZDs and GLP-1RAs had higher SMI and handgrip strength than the ones who were not using these drugs (all $p<0.05$, Fig. 1A, C). Moreover, in women, sulfonylureas were associated with lower SMI, and glinides were associated with lower handgrip strength, and gait speed $(p<0.05$, Fig. 1D, F). There were no differences of muscle mass and function between patients using and not using metformin and SGLT2 inhibitors (all $p>0.05$, Fig. 1).

We also compared the SMI, handgrip strength, and gait speed among drug-naïve patients, and patients treated with insulin, metformin, sulfonylureas, or acarbose monotherapy. The sample sizes of patients treated with glinides, TZDs, DPP4 inhibitors, SGLT2 inhibitors, and GLP-1RAs monotherapy were too small for analysis $(n<10)$. The Kruskal-Wallis test showed that the acarbose group had the lowest SMI and handgrip strength $\left[6.81(6.52,7.11) \mathrm{kg} / \mathrm{m}^{2}\right.$ and 22.54 $(19.28,25.79) \mathrm{kg}, p=0.028$ and 0.001 , respectively]. The SMI and handgrip strength were significantly lower in the acarbose group than in the metformin group and drug-naïve group (both $p<0.05$, Fig. 2A, B), Insulin group also had lower handgrip strength compared with the drug-naïve group (Fig. 2B).

We also performed propensity score matching to adjust the confounders (age, gender, BMI, HbA1c, and duration of diabetes) which may 

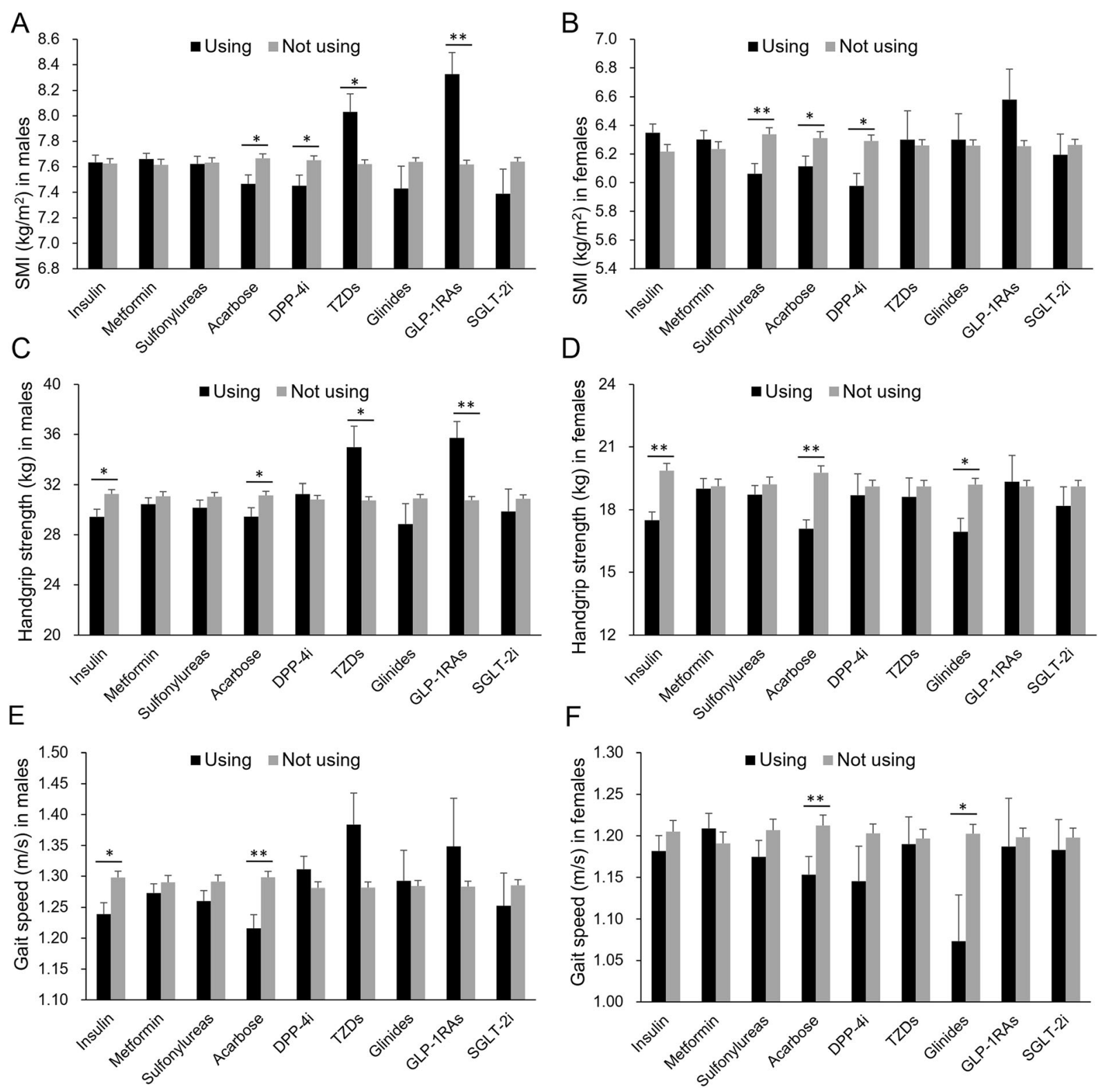

Fig. 1 Muscle mass and function in patients treated with glucose-lowering drugs. A Skeletal muscle index (SMI) in men treated with insulin $(n=151)$, metformin $(n=221)$, sulfonylureas $(n=143)$, acarbose $(n=110)$, dipeptidyl peptidase 4 (DPP 4$)$ inhibitors $(n=64)$, TZDs $(n=16)$, glinides $(n=21)$, GLP-1RAs $(n=12)$, and SGLT2 inhibitors $(n=23)$ (black bars) or not (gray bars); B SMI in women treated with insulin $(n=136)$,

metformin $(n=144)$, sulfonylureas $(n=116)$, acarbose $(n=105)$, DPP4 inhibitors $(n=40)$, TZDs $(n=10)$, glinides $(n=19)$, GLP-1RAs $(n=10)$, and SGLT2 inhibitors $(n=17)$ or not. C, D Handgrip strength in men and women; $\mathbf{E}, \mathbf{F}$ gait speed in men and women. ${ }^{*} p<0.05,{ }^{* *} p<0.01$, using vs. not using. Data are mean (SEM)

influence both muscles and the choice of glucose-lowering drugs. A total of 208 matched patients who were not using acarbose therapy (drug-naïve patients and patients using glucose-

lowering drugs except acarbose) were included in the propensity score matching, and the SMI and gait speed remained significantly lower in 

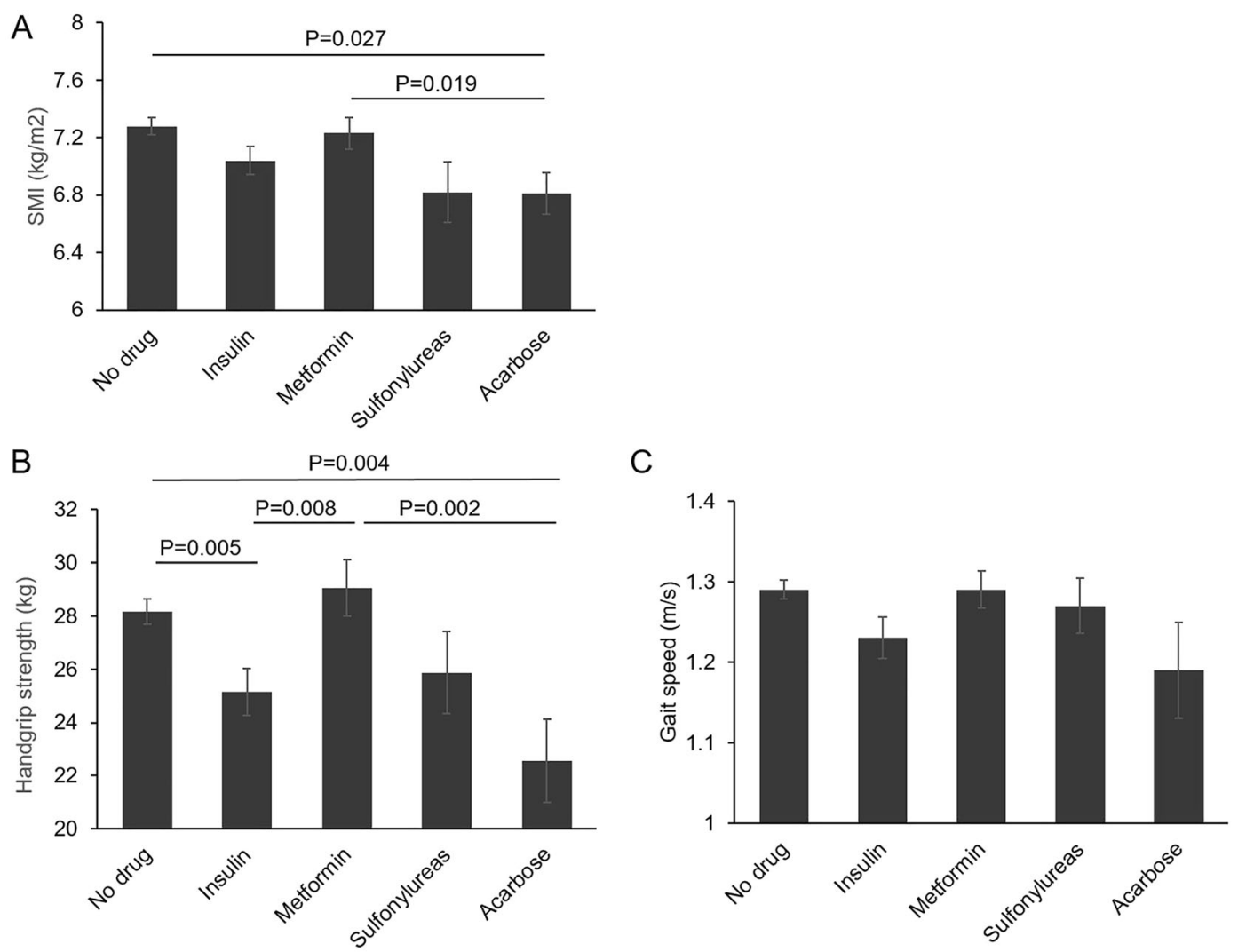

Fig. 2 Muscle mass and function in drug-naïve patients, and patients treated with insulin, metformin, sulfonylureas, or acarbose monotherapy. A Skeletal muscle index (SMI), $\mathbf{B}$ handgrip strength, $\mathbf{C}$ gait speed in drug-naïve patients

$(n=327)$, and patients treated with insulin $(n=103)$, metformin $(n=71)$, sulfonylureas $(n=37)$, or acarbose $(n=22)$ monotherapy. Data are mean (SEM)
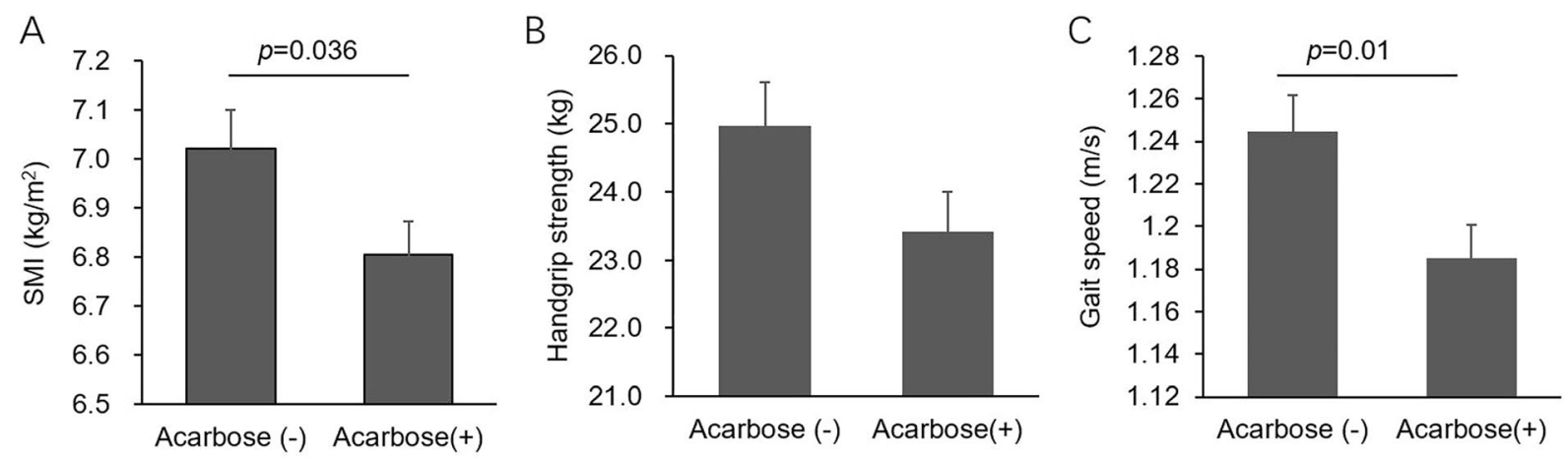

Fig. 3 Muscle mass and function in patients using acarbose after propensity score matching. A Skeletal muscle index (SMI), B handgrip strength, $\mathbf{C}$ gait speed in patients using acarbose $(n=215)$ and propensity score matched patients without acarbose therapy $(n=208)$. Age, gender, BMI, HbAlc, and duration of diabetes were adjusted in the propensity score matching analysis. Data are mean (SEM) 
patients with acarbose compared to the controls (both $p<0.05$, Fig. 3).

\section{Muscle Mass and Function and Glucose- Lowering Drugs in Old Patients}

After the age stratification, SMI, handgrip strength, and gait speed remain lower in old patients ( $\geq 60$ years) using acarbose compared with the other old patients (all $p<0.05$, Fig. 4). In the insulin group, old patients had increased SMI and decrease handgrip strength compared with old patients who were not using insulin (Fig. 4). Old patients using DPP4 inhibitors had lower SMI, and similar handgrip strength and gait speed compared with the other elderly (Fig. 4). The proportions of sarcopenia were $20.75 \%, 26.14 \%, 26.71 \%$, and $30.50 \%$ in

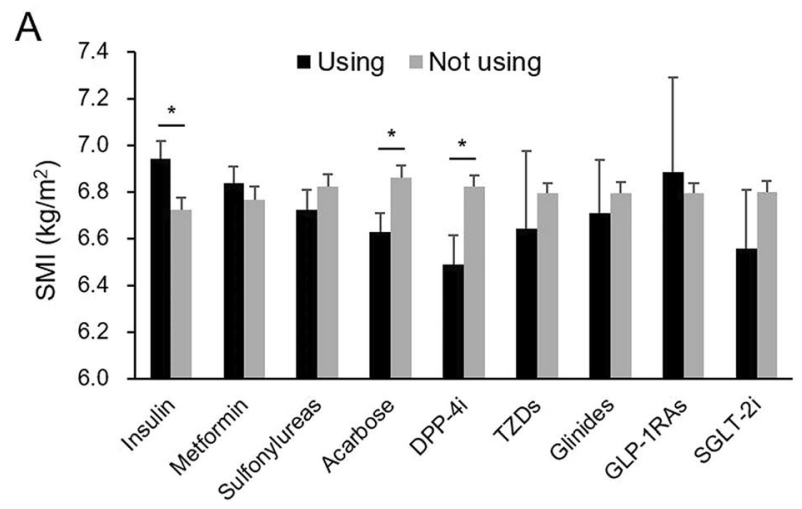

B

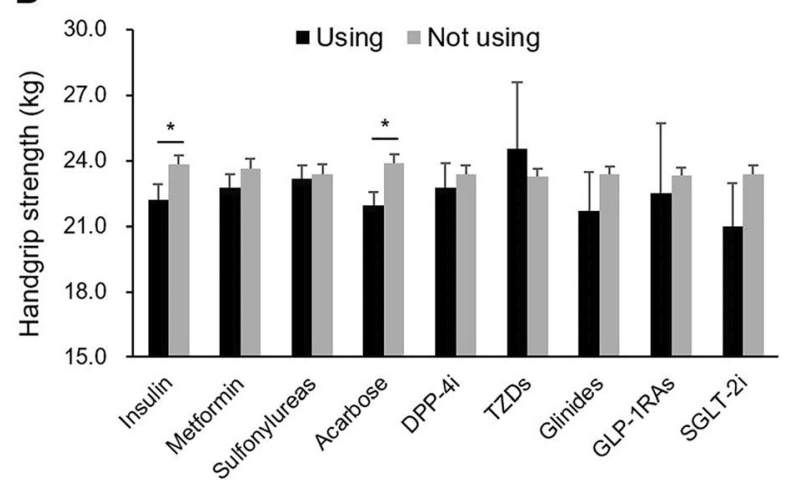

Fig. 4 Muscle mass and function in old patients ( $\geq 60$ years). A Skeletal muscle index (SMI), B handgrip strength,C gait speed in patients treated with insulin ( $n=159)$, metformin $(n=176)$, sulfonylureas $(n=161)$, acarbose $(n=141)$, dipeptidyl peptidase 4 (DPP4) patients using insulin, metformin, sulfonylureas, and acarbose, respectively (insulin vs. acarbose, $p=0.063)$. The proportions of severe sarcopenia (patients who had both low muscle strength and low physical performance) in patients using insulin, metformin, sulfonylureas, and acarbose were $4.40 \%, 8.52 \%$, $10.56 \%$, and $11.35 \%$ (insulin vs. acarbose, $p=0.031)$.

\section{Characteristics of Patients Using Acarbose}

In the present study, $20.6 \%$ patients were using acarbose, and the proportion rose to $28.7 \%$ in elderly patients. The patients using acarbose were older, and had longer diabetes duration, more women, lower weight, BMI, and waist to hip ratio, lower albumin, blood lipids,

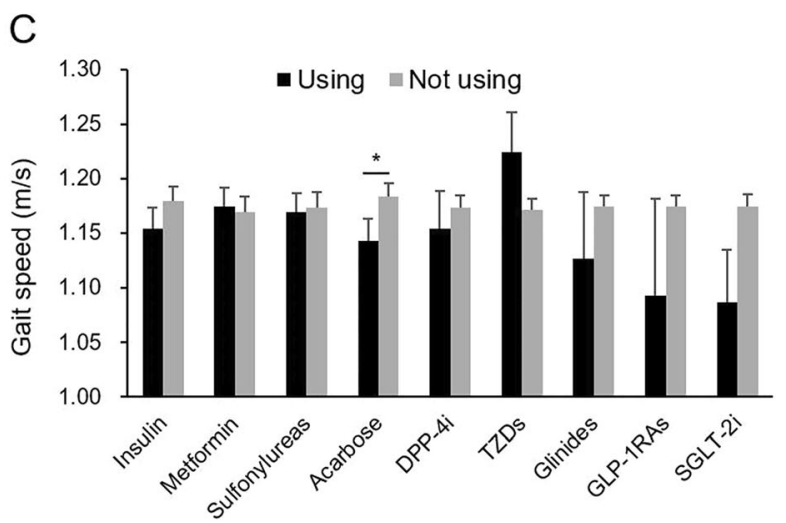

inhibitors $(n=50)$, TZDs $(n=9)$, glinides $(n=21)$, GLP-1RAs $(n=7)$, and SGLT2 inhibitors $(n=17)$ (black bars) or not (gray bars). ${ }^{*} p<0.05$, using vs. not using. Data are mean (SEM) 


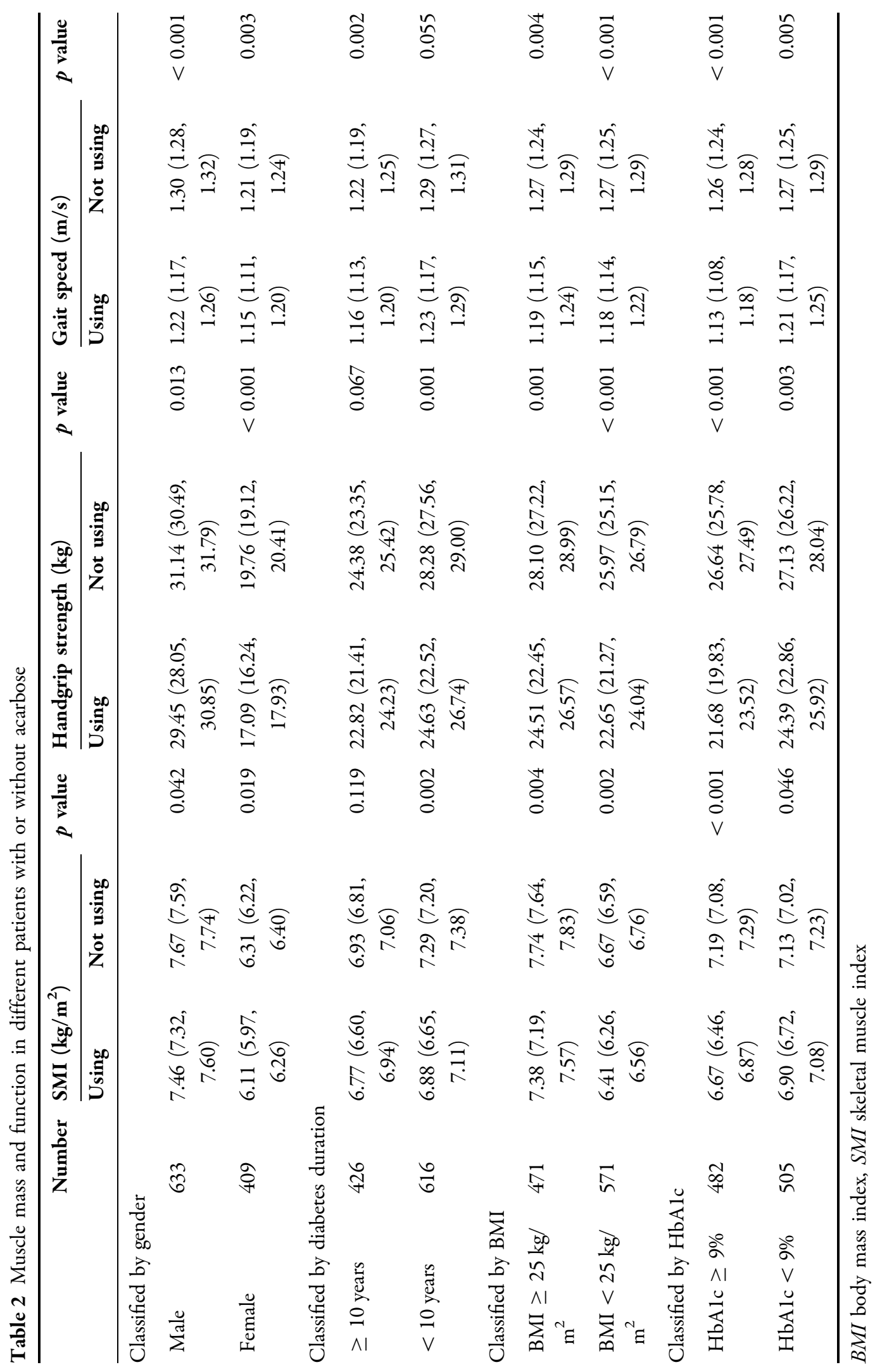


hemoglobin, and HbA1c levels compared with the other patients $(p$ all $<0.01$, Supplementary Table 1). As diabetes duration, gender, body weight, and blood glucose control may influence the prescription of acarbose, we stratified patients according to these characteristics for further analysis. After the stratification of diabetes duration, gender, BMI, and $\mathrm{HbA1c}$, patients who were treated with acarbose still had lower SMI, handgrip strength, and gait speed compared with the patients who were not using acarbose in each group (all $p<0.05$ ), except the SMI and handgrip strength in patients with a long diabetes duration ( $\geq 10$ years) (Table 2 ).

\section{DISCUSSION}

The present study showed a significant lower muscle mass and function in patients taking acarbose both as monotherapy and in combination compared with the other patients. As far as we are aware, there has not been a study comparing the muscle mass and function among so many different kinds of glucose-lowering drugs treatment directly before, and the effect of acarbose on muscles has not been reported yet [11].

Acarbose is commonly used in patients with T2DM. Previous study found that acarbose is similar to metformin in efficacy, and is considered as a viable choice for initial therapy in Chinese patients newly diagnosed with T2DM $[32,33]$. However, our study showed that acarbose, rather than metformin, may be associated with low muscle mass and function. Among insulin, metformin, sulfonylureas, acarbose, DPP4 inhibitors, TZDs, glinides, GLP-1RAs, and SGLT2 inhibitors, acarbose was the only glucose-lowering drug associated with both low muscle mass and low muscle function, and was also the only one that has never been reported in previous studies of muscles as far as we aware of $[6,21,22]$. Moreover, the association between acarbose and muscle mass/function was independent of age, gender, BMI, HbA1c, and duration of diabetes. Whatever the causal relationship between the use of acarbose and decreased muscle mass and function, the assessment of muscles should be taken more seriously in patients using acarbose. Moreover, the risk of sarcopenia in patients using acarbose needs to be studied further.

The association between acarbose and low muscle mass and function should not be totally unexpected, as acarbose is an $\alpha$-glucosidase inhibitor that inhibits the digestion and absorption of carbohydrates in the small intestine. This effect of acarbose on absorption of carbohydrates leads to reduction of energy, especially in Asia where rice forms a major component of the diet and the dietary contribution of carbohydrate is high [33, 34]. Acarbose also alters gut microbiota [35]. Gut microbiota affects the metabolism of carbohydrate and short-chain fatty acids [36], and may act as a mediator of the effects of nutrition on muscle cells [37]. In the present study, patients who were treated with acarbose had lower blood lipid levels, weight, albumin, and hemoglobin, which was also observed in patients with sarcopenia, and were all indicators of nutrition status.

Acarbose and SGLT2 inhibitors were the only two kinds of antidiabetic drugs that mainly lead to energy loss from the body, while the other drugs commonly regulate the transfer of energy inside and outside the cells. SGLT2 inhibitors, which induced glycosuria and energy loss from kidney, have also been shown to reduce muscle mass in previous studies [38, 39]. In the present study, the SMI, handgrip strength, and gait speed levels in patients using SGLT2 inhibitors were lower than those in the others, especially in elderly patients. However, the differences were not statistically significant, which may be because of the small sample size. The effects of SGLT2 inhibitors on muscle remain controversial. Hirata et al. found that hyperglycemia directly exerted catabolic effects on skeletal muscle and promoted muscle atrophy via a WWP1/KLF15 pathway. They also found that administration of empagliflozin lowered the blood glucose concentration and prevented the reduction in skeletal muscle mass [40].

Not only empagliflozin but also other glucose-lowering drugs may prevent muscle atrophy via reducing blood glucose. The hypoglycemic effect may mask the direct effects 
of these drugs on muscles. As a result, the association between most glucose-lowering drugs, such as insulin, metformin, and GLP1RAs, and muscle mass and function was unclear and even controversial [21]. However, the hypoglycemic effect of acarbose cannot mask its negative effects on muscles. In the present study, stable hypoglycemic treatment and poor glycemic control in the last 3 months partially diminished the influence of blood glucose change on muscles caused by drugs, which also contributed to the similar HbA1c and HOMA2-IR levels in patients with and without sarcopenia.

Bouchi et al. showed that insulin treatment could attenuate the progression of sarcopenia in patients with type 2 diabetes [41]. We also found increased SMI in elderly patients using insulin therapy. The result was similar to their study. However, handgrip strength and gait speed were decreased in these patients.

DPP4 inhibitors were associated with decreased SMI but not decreased handgrip strength and gait speed in the present study. However, the patients using GLP-1RAs had quite a different characteristic muscle mass, especially in men, although DPP4 inhibitors increase plasma GLP-1 levels and have a similar mechanism of hypoglycemic effect as GLP1RAs. Cetrone et al. also suggested that GLP1RAs showed beneficial effects in muscles but their effects on the age-dependent muscle atrophy remained unknown [21]. The sample size of patients using DPP4 inhibitors and GLP1RAs was small, and further study about the effects of these kinds of drugs on muscles is needed.

Our study found increased muscle mass and strength in men using TZDs, and partially provided evidence to resolve the controversy around the effectiveness of PPAR agonists in muscle atrophy [21].

One limitation of our study was that the cross-sectional study could not explain the causality between the use of blood glucoselowering drugs and the change of muscle mass and function. Other factors, such as duration of diabetes, age, weight, and blood lipid and glucose levels, may simultaneously affect the drug prescription and muscles. Moreover, the distribution of patients using different drugs was lopsided, and the sample size was quite small for patients using glinides, TZDs, DPP4 inhibitors, SGLT2 inhibitors, and GLP-1RAs. Therefore, the results about these drugs were unreliable.

\section{CONCLUSIONS}

Our data suggest that patients with T2DM treated with acarbose may be at high risk for decreased skeletal muscle mass and strength compared with those who do not receive acarbose treatment. Whether the administration of acarbose influences the incident sarcopenia in randomized controlled trials remains to be elucidated. Assessment and exercise of muscles in patients with long-term acarbose treatment should be considered.

\section{ACKNOWLEDGEMENTS}

We thank the members of the endocrinology department of Nanjing First Hospital for their support.

Funding. This work was supported by the National Key R\&D Program of China (No. 2018YFC1314103), and the fellowship of China Postdoctoral Science Foundation (No. 2020M671535). No funding was received from the journal for the publication of this article.

Authorship. All named authors meet the International Committee of Medical JournalEditors (ICMJE) criteria for authorship for this article, take responsibility for the integrity of the work as a whole, and have given their approval for this version to be published.

Authors' Contributions. JHM and $\mathrm{YH}$ are responsible for the conception and design of the study. LLJ and YH carried out statistical analysis and wrote the first draft of the manuscript. LLJ, XHX, MHL, BD, and RNY contributed to provide clinical samples and acquisition of data. HYW approved the final version of the 
manuscript. JHM and $\mathrm{YH}$ contributed to obtain funding, and are the guarantors of this work. All authors revised the manuscript critically for important intellectual content.

Disclosures. Lan-lan Jiang, Xiao-hua $\mathrm{Xu}$, Meng-hui Luo, Hui-ying Wang, Bo Ding, Rengna Yan, Yun Hu and Jian-hua Ma have declared that no competing interests exist.

Compliance with Ethics Guidelines. This was a retrospective, cross-sectional study, and was approved by the Institutional Ethical Committee of Nanjing First Hospital, Nanjing Medical University (20160201-01). The methods were conducted in accordance with the Declaration of Helsinki guidelines, including any relevant details. The need for informed consent was waived for the present study as the research involves no more than minimal risk to subjects, and the waiver did not adversely affect the rights and welfare of the subjects. All data was retrospectively collected from the medical review database without involving any identifiable private information under the consent of the corresponding department.

Data Availability. The datasets used and/or analyzed during the current study are available from the corresponding author on reasonable request.

Open Access. This article is licensed under a Creative Commons Attribution-NonCommercial 4.0 International License, which permits any non-commercial use, sharing, adaptation, distribution and reproduction in any medium or format, as long as you give appropriate credit to the original author(s) and the source, provide a link to the Creative Commons licence, and indicate if changes were made. The images or other third party material in this article are included in the article's Creative Commons licence, unless indicated otherwise in a credit line to the material. If material is not included in the article's Creative Commons licence and your intended use is not permitted by statutory regulation or exceeds the permitted use, you will need to obtain permission directly from the copyright holder. To view a copy of this licence, visit http://creativecommons.org/licenses/by$\mathrm{nc} / 4.0 /$.

\section{REFERENCES}

1. Beaudart C, Rizzoli R, Bruyere O, Reginster JY, Biver E. Sarcopenia: burden and challenges for public health. Arch Public Health. 2014;72(1):45. https:// doi.org/10.1186/2049-3258-72-45.

2. Guralnik JM, Ferrucci L, Pieper CF, et al. Lower extremity function and subsequent disability: consistency across studies, predictive models, and value of gait speed alone compared with the short physical performance battery. J Gerontol A Biol Sci Med Sci. 2000;55(4):M221-31. https://doi.org/10.1093/ gerona/55.4.m221.

3. Vetrano DL, Landi F, Volpato S, et al. Association of sarcopenia with short- and long-term mortality in older adults admitted to acute care wards: results from the CRIME study. J Gerontol A Biol Sci Med Sci. 2014;69(9):1154-61. https://doi.org/10.1093/ gerona/glu034.

4. Edwards MH, Dennison EM, Aihie Sayer A, Fielding $\mathrm{R}$, Cooper C. Osteoporosis and sarcopenia in older age. Bone. 2015;80:126-30. https://doi.org/10. 1016/j.bone.2015.04.016.

5. Mijnarends DM, Luiking YC, Halfens RJG, et al. Muscle, health and costs: a glance at their relationship. J Nutr Health Aging. 2018;22(7):766-73. https://doi.org/10.1007/s12603-018-1058-9.

6. Mesinovic J, Zengin A, De Courten B, Ebeling PR, Scott D. Sarcopenia and type 2 diabetes mellitus: a bidirectional relationship. Diabetes Metab Syndr Obes. 2019;12:1057-72. https://doi.org/10.2147/ DMSO.S186600.

7. Wang T, Feng X, Zhou J, et al. Type 2 diabetes mellitus is associated with increased risks of sarcopenia and pre-sarcopenia in Chinese elderly. Sci Rep. 2016;6:38937. https://doi.org/10.1038/ srep38937.

8. Fung FY, Koh YLE, Malhotra R, et al. Prevalence of and factors associated with sarcopenia among multi-ethnic ambulatory older Asians with type 2 diabetes mellitus in a primary care setting. BMC Geriatr. 2019;19(1):122. https://doi.org/10.1186/ s12877-019-1137-8.

9. Bouchi R, Fukuda T, Takeuchi T, et al. Association of sarcopenia with both latent autoimmune diabetes in adults and type 2 diabetes: a cross-sectional 
study. J Diabetes Complicat. 2017;31(6):992-6. https://doi.org/10.1016/j.jdiacomp.2017.02.021.

10. Murata Y, Kadoya Y, Yamada S, Sanke T. Sarcopenia in elderly patients with type 2 diabetes mellitus: prevalence and related clinical factors. Diabetol Int. 2018;9(2):136-42. https://doi.org/10.1007/s13340017-0339-6.

11. Izzo A, Massimino E, Riccardi G, Della PG. A narrative review on sarcopenia in type 2 diabetes mellitus: prevalence and associated factors. Nutrients. 2021. https://doi.org/10.3390/nu13010183.

12. Tournadre A, Vial G, Capel F, Soubrier M, Boirie Y. Sarcopenia. Jt Bone Spine. 2019;86(3):309-14. https://doi.org/10.1016/j.jbspin.2018.08.001.

13. Semba RD, Bandinelli S, Sun K, Guralnik JM, Ferrucci L. Relationship of an advanced glycation end product, plasma carboxymethyl-lysine, with slow walking speed in older adults: the InCHIANTI study. Eur J Appl Physiol. 2010;108(1):191-5. https://doi.org/10.1007/s00421-009-1192-5.

14. Patti AM, Rizvi AA, Giglio RV, Stoian AP, Ligi D, Mannello F. Impact of glucose-lowering medications on cardiovascular and metabolic risk in type 2 diabetes. J Clin Med. 2020. https://doi.org/10.3390/ jcm9040912.

15. Tsunosue M, Mashiko N, Ohta Y, et al. An alphaglucosidase inhibitor, acarbose treatment decreases serum levels of glyceraldehyde-derived advanced glycation end products (AGEs) in patients with type 2 diabetes. Clin Exp Med. 2010;10(2):139-41. https://doi.org/10.1007/s10238-009-0074-9.

16. American Diabetes Association. 9. Pharmacologic approaches to glycemic treatment: standards of medical care in diabetes-2020. Diabetes Care. 2020;43(Suppl 1):S98-110. https://doi.org/10.2337/ dc20-S009.

17. Wrobel MP, Marek B, Kajdaniuk D, Rokicka D, Szymborska-Kajanek A, Strojek K. Metformin: a new old drug. Endokrynol Pol. 2017;68(4):482-96. https://doi.org/10.5603/EP.2017.0050.

18. Lebovitz HE. Thiazolidinediones: the forgotten diabetes medications. Curr Diabetes Rep. 2019;19(12):151. https://doi.org/10.1007/s11892019-1270-y.

19. Lee CG, Boyko EJ, Barrett-Connor E, et al. Insulin sensitizers may attenuate lean mass loss in older men with diabetes. Diabetes Care. 2011;34(11): 2381-6. https://doi.org/10.2337/dc11-1032.

20. Miller SL, Wolfe RR. The danger of weight loss in the elderly. J Nutr Health Aging. 2008;12(7): 487-91. https://doi.org/10.1007/BF02982710.
21. Cetrone M, Mele A, Tricarico D. Effects of the antidiabetic drugs on the age-related atrophy and sarcopenia associated with diabetes type II. Curr Diabetes Rev. 2014;10(4):231-7. https://doi.org/10. 2174/1573399810666140918121022.

22. Cui M, Gang X, Wang G, et al. A cross-sectional study: associations between sarcopenia and clinical characteristics of patients with type 2 diabetes. Medicine (Baltimore). 2020;99(2): e18708. https:// doi.org/10.1097/MD.0000000000018708.

23. Aghili R, Malek M, Valojerdi AE, Banazadeh Z, Najafi L, Khamseh ME. Body composition in adults with newly diagnosed type 2 diabetes: effects of metformin. J Diabetes Metab Disord. 2014;13(1):88. https://doi.org/10.1186/s40200-014-0088-z.

24. Hamaguchi Y, Kaido T, Okumura S, et al. Proposal for new diagnostic criteria for low skeletal muscle mass based on computed tomography imaging in Asian adults. Nutrition. 2016;32(11-12):1200-5. https://doi.org/10.1016/j.nut.2016.04.003.

25. Pagotto V, Silveira EA. Methods, diagnostic criteria, cutoff points, and prevalence of sarcopenia among older people. Sci World J. 2014;2014: 231312. https://doi.org/10.1155/2014/231312.

26. Dabak MR, Sevinc E, Tuzun S, Gun EO. Evaluation of muscle mass in obesity, prediabetes and diabetes mellitus by different equations used for the measurement of muscle mass. Diabetes Metab Syndr. 2019;13(3):2148-51. https://doi.org/10.1016/j.dsx. 2019.05.007.

27. Ding B, Sun R, Zhai XF, et al. Association of high circulating testosterone with increased glycaemic variability in type 2 diabetes: a cross-sectional study in China. Diabetes Metab Res Rev. 2019;35(4): e3126. https://doi.org/10.1002/dmrr.3126.

28. Jia W, Weng J, Zhu D, et al. Standards of medical care for type 2 diabetes in China 2019. Diabetes Metab Res Rev. 2019;35(6): e3158. https://doi.org/ 10.1002/dmrr.3158.

29. Chinese Society of Osteoporosis and Bone Mineral Research CMA. A guide to diagnosis and treatment of primary osteoporosis. Chin J Osteoporos Bone Miner Res. 2017;10(5):413-44. https://doi.org/10. 3969/j.issn.1674-2591.2017.05.002.

30. Duvivier BM, Schaper NC, Hesselink MK, et al. Breaking sitting with light activities vs structured exercise: a randomised crossover study demonstrating benefits for glycaemic control and insulin sensitivity in type 2 diabetes. Diabetologia. 2017;60(3):490-8.

31. Chen LK, Woo J, Assantachai P, et al. Asian Working Group for Sarcopenia: 2019 consensus update 
on sarcopenia diagnosis and treatment. J Am Med Dir Assoc. 2020;21(3):300-7. https://doi.org/10. 1016/j.jamda.2019.12.012.

32. Yang W, Liu J, Shan Z, et al. Acarbose compared with metformin as initial therapy in patients with newly diagnosed type 2 diabetes: an open-label, non-inferiority randomised trial. Lancet Diabetes Endocrinol. 2014;2(1):46-55. https://doi.org/10. 1016/S2213-8587(13)70021-4.

33. Ma RC. Acarbose: an alternative to metformin for first-line treatment in type 2 diabetes? Lancet Diabetes Endocrinol. 2014;2(1):6-7. https://doi.org/10. 1016/S2213-8587(13)70107-4.

34. Zhu Q, Tong Y, Wu T, Li J, Tong N. Comparison of the hypoglycemic effect of acarbose monotherapy in patients with type 2 diabetes mellitus consuming an Eastern or Western diet: a systematic metaanalysis. Clin Ther. 2013;35(6):880-99. https://doi. org/10.1016/j.clinthera.2013.03.020.

35. Zhang M, Feng R, Yang M, et al. Effects of metformin, acarbose, and sitagliptin monotherapy on gut microbiota in Zucker diabetic fatty rats. BMJ Open Diabetes Res Care. 2019;7(1): e000717. https://doi.org/10.1136/bmjdrc-2019-000717.

36. Kyriachenko Y, Falalyeyeva T, Korotkyi O, Molochek N, Kobyliak N. Crosstalk between gut microbiota and antidiabetic drug action. World J Diabetes. 2019;10(3):154-68. https://doi.org/10. 4239/wjd.v10.i3.154.
37. Ticinesi A, Lauretani F, Milani C, et al. Aging gut microbiota at the cross-road between nutrition, physical frailty, and sarcopenia: is there a gutmuscle axis? Nutrients. 2017. https://doi.org/10. 3390/nu9121303.

38. Sasaki T, Sugawara M, Fukuda M. Sodium-glucose cotransporter 2 inhibitor-induced changes in body composition and simultaneous changes in metabolic profile: 52-week prospective LIGHT (Luseogliflozin: the Components of Weight Loss in Japanese Patients with Type 2 Diabetes Mellitus) Study. J Diabetes Investig. 2019;10(1):108-17. https://doi.org/10.1111/jdi.12851.

39. Nagai Y, Fukuda H, Kawanabe S, Nakagawa T, Ohta A, Tanaka Y. Differing effect of the sodium-glucose cotransporter 2 inhibitor ipragliflozin on the decrease of fat mass vs. lean mass in patients with or without metformin therapy. J Clin Med Res. 2019;11(4):297-300. https://doi.org/10.14740/ jocmr3785.

40. Hirata Y, Nomura K, Senga Y, et al. Hyperglycemia induces skeletal muscle atrophy via a WWP1/KLF15 axis. JCI Insight. 2019. https://doi.org/10.1172/jci. insight. 124952.

41. Bouchi R, Fukuda T, Takeuchi $\mathrm{T}$, et al. Insulin treatment attenuates decline of muscle mass in Japanese patients with type 2 diabetes. Calcif Tissue Int. 2017;101(1):1-8. https://doi.org/10.1007/ s00223-017-0251-X. 